\title{
$1 \quad$ Topical cream with live lactobacilli modulates the skin microbiome and reduce acne symptoms
}

2 Sarah Lebeer ${ }^{1^{*}}$, Eline Oerlemans $^{1^{*}},{\text { Ingmar } \text { Claes }^{1^{*}}, \text { Sander Wuyts }^{1}, \text { Tim Henkens }}^{2}$, Irina Spacova ${ }^{1}$,

3 Marianne van den Broek ${ }^{1}$, Ines Tuyaerts ${ }^{1}$, Stijn Wittouck ${ }^{1}$, Ilke De Boeck ${ }^{1}$, Camille N. Allonsius ${ }^{1}$, Filip

$4 \quad$ Kiekens ${ }^{2}$, Julien Lambert ${ }^{3}$

$5 \quad$ *Equal contribution

6

$7{ }^{1}$ University of Antwerp, Department of Bioscience Engineering, Groenenborgerlaan 171, B-2020

8 Antwerp, Belgium.

9 'University of Antwerp, Department of Pharmaceutical, Biomedical and Veterinary Sciences,

10 Laboratory of Pharmaceutical Technology and Biopharmacy, Universiteitsplein 1, B-2610 Wilrijk,

11 Belgium.

$12{ }^{3}$ University Hospital Antwerp/University of Antwerp Department of Dermatology and Venereology,

13 Wilrijkstraat 10, 2650 Edegem, Belgium.

14 Correspondence: Sarah Lebeer, University of Antwerp, Department of Bioscience Engineering,

15 Groenenborgerlaan 171, B-2020 Antwerp, Belgium. Telephone: +3232653285 E-mail:

16 sarah.lebeer@uantwerpen.be

19 The skin is home to an important part of our commensal microbiota, despite it being a cool, acidic

20 and desiccated environment. Tailored microbiome modulation approaches with, for example

21 probiotics, are highly challenging for this body site. Here we show by next-generating sequencing

22 that Lactobacillus taxa -especially those known to be dominant in the human vagina- are

23 underestimated members of the skin microbiota. Specific Lactobacillus strains were selected in the 
lab and formulated in a viable form in an oil in water-based topical cream. Facial application by patients with mild-to-moderate acne symptoms was able to reduce inflammatory lesions and comedone formation. This was associated with a temporary modulation of the skin microbiome,

27 including a reduction in relative abundance of staphylococci and an increase in lactobacilli. Skin microbiome modulation by addition of carefully formulated lactobacilli seems to be new therapeutic option to reduce antibiotic use for common acne symptoms.

\section{Introduction}

32 Being the most extensive interface of the human body with the environment, the skin acts as a home

33 to an important part of our commensal microbiota. Similar to the gut, the skin microbiota have 34 essential roles in the education of our immune system and the protection against invading pathogens and other foreign substances. With recent advances in DNA sequencing approaches, our knowledge has been improved on the biogeography of the skin microbiota at different body sites ${ }^{1}$. We are now

37 transitioning from these descriptive, observational studies towards a better understanding of the

38 functional roles of the commensal microbiota, allowing the design of tailored modulation

39 approaches. However, compared with the richer environment of our intestines, the skin lacks many 40 nutrients beyond basic proteins and lipids, with sweat, sebum and the stratum corneum being main 41 resources ${ }^{2}$. In addition, the skin is a cool, acidic and desiccated environment and skin cells are

42 frequently renewed and shed, so that strategies targeting the skin microbiome are highly 43 challenging. For example, probiotics, i.e. live micro-organisms that, when applied in adequate 44 amounts, promote a health effect on the host ${ }^{3}$, have not yet been widely considered for direct 45 application on the skin.

46 One of the most common skin diseases is acne vulgaris, a chronic inflammatory skin condition of the 47 sebaceous follicles and glands. The pathogenesis of acne vulgaris is multifactorial, with increased 48 sebum production, alteration in the quality of sebum lipids, dysregulation of the hormone 
environment and follicular hyperkeratinization as contributing factors. In addition, specific strains of

50 the facultative anaerobe Cutibacterium acnes (formerly known as Propionibacterium acnes ${ }^{4}$ ) are

51 involved in the inflammation of the skin, especially by secreting lipase enzymes that are able to

52 metabolize sebum into free fatty acids which may lead to skin irritation ${ }^{5}$. Yet, the observation that

53 almost all adults are colonized with $C$. acnes but only a minority have acne, highlights that other

54 bacteria such as Staphylococcus species can be linked to acne pathogenesis as pathobionts or disease

55 modulators $^{6}$. Therefore, both oral and topical antibiotics such as doxycycline, minocycline and

56 clindamycin are frequently used by acne patients ${ }^{7}$, but because of rising problems of antibiotic

57 resistance, various alternative therapies need to be developed ${ }^{8}$.

58 Here we explored the potential of topically applied, live probiotic lactobacilli to beneficially modulate

59 cutaneous microbial interactions and host inflammatory responses in subjects with mild-to-moderate

60 acne symptoms. Lactobacilli were selected based on their long history of safe use in fermented

61 foods $^{9}$, the gastro-intestinal ${ }^{10}$, urogenital tract ${ }^{11}$ and nasal cavity ${ }^{12}$, but it was unsure whether

62 lactobacilli could also thrive and have health-promoting activities on the skin.

\section{Results and discussion}

64 Prevalence of Lactobacillus on the skin. Because lactobacilli are not considered to be commensals of

65 the skin, we first monitored the prevalence of lactobacilli on the skin of healthy volunteers. Their

66 relative abundance was explored through 165 amplicon sequencing via Illumina MiSeq (separate runs

67 for V1V2 and V4 variable regions) of facial skin samples (cheek) of 30 volunteers (15 male and 15

68 female), who did not display acne-related symptoms. In the samples of all female volunteers and 12

69 male volunteers Lactobacillus sequences were found (Figure 1a). Lactobacillus species generally did

70 not occur in the top five of most abundant taxa present on the skin. However, some volunteers

71 showed a relative high abundance of Lactobacillus taxa (amplicon sequence variants or ASVs), up to

$726.4 \%$ (based on V1V2 $16 \mathrm{~S}$ sequencing) or $14.3 \%$ (by V4 $16 \mathrm{~S}$ sequencing) (Figure 1b, Extended data

73 Figure 1a-b). The relative abundance of Lactobacillus taxa based on both runs (V1V2 and V4) was 

also 10 -fold higher in women compared to men, with an average relative abundance of $0.8 \%(1.4 \%$ in women and $0.2 \%$ in men, Kruskal-Wallis $p=0.0005)$. (Extended Data Figure $1 \mathrm{~b})$. Lactobacillus taxa can thus be considered as endogenous members of the skin microbiota, although their relative abundance is lower than Staphylococcus, Corynebacterium, Cutibacterium (often still classified as Propionibacterium), and Streptococcus, which were the most the dominant taxa in our dataset for both variable regions sequenced (Extended Data figure 1 a).

To confirm our in-house generated data and investigate whether our results are facial site-specific, the presence of lactobacilli was also substantiated in publicly available skin metagenome shotgun datasets by using the curatedMetagenomicData R-package recently described by Pasolli et al. ${ }^{13}$. In total, 466 samples from three different studies ${ }^{14,15}$ and 16 were analyzed. Of these samples, 38\% (177/466) showed the presence of at least one Lactobacillus species (Figure 1a), but only 29 samples showed a relative abundance higher than $1 \%$. Yet, high relative abundances up to $52 \%$ on the skin were also observed (average relative abundance based on curated metagenomics was $3.79 \%$ ) (Figure 1b). We also included $16 \mathrm{~S}$ amplicon data from the Human Microbiome Project (V3V5) ${ }^{17}$ where the relative abundance was $24.9 \%$ on average due to some outliers having up to $90 \%$ relative abundance (Figure 1b). The relative abundance of Lactobacillus sequences in the skin samples was also compared to the publicly available data of other human body sites (both $16 \mathrm{~S}$ and curated metagenome) (Figure 1b). As expected, the vagina showed the highest relative abundance of Lactobacillus taxa, but the skin turned out to be the second most important niche for these taxa. Moreover, to have a better idea of the phylogenetic diversity of all Lactobacillus taxa present, we also plotted all data on a phylogenetic tree of the Lactobacillus genus complex ${ }^{18}$ (Figure 1c). These data indicate that taxa typically associated with the human vagina, Lactobacillus crispatus, $L$. iners, $L$. gasseri and L. jensenii were also found as the most prevalent lactobacilli on the skin (Figure $1 \mathbf{d}$ and 1e). Also members of the more niche-flexible Lactobacillus taxa ${ }^{19}$, i.e. from the $L$. plantarum $/ L$. pentosus group and L. casei/paracasei/rhamnosus group, were frequently detected (Figure 1d and 1e). The occurrence of Lactobacillus taxa on the skin is in agreement with the fact that after normal 
delivery through the birth canal, these bacteria originating from the mother are among the first to colonize neonate $\operatorname{skin}^{20}$. The data presented here (Figure 1 a-e) indicate that these Lactobacillus taxa are still present in adults, but do not stay dominant in the different human body skin sites studied. Yet, despite their low relative abundance, they could still play a role as keystone microbes, recently redefined as taxa exerting a considerable influence on microbiome structure and functioning irrespective of their abundance across space and time $\mathrm{e}^{21}$. Therefore, we subsequently aimed to manipulate biotic interactions of lactobacilli on the skin.

Rationale in vitro strain selection. Lactobacillus strains were selected from our in-house available laboratory collection (Extended Data Table 1) for tailored application in patients with mild-to moderate acne symptoms. A thorough screening approach was applied based on the rationalization that the strains had to be safe, applicable (being robust and showing niche-flexibility as described for lactobacilli by Duar et $a I^{19}$ ) and have the capacity to exert the desired beneficial functions on the human skin including microbiome modulation, immune modulation and epithelial barrier enhancement (Figure 2a). Key properties were substantiated with laboratory tests, genome screening and information available in the literature. Three Lactobacillus strains were selected i.e. Lactobacillus rhamnosus GG, Lactobacillus plantarum WCFS1 and L. pentosus KCA1. The rationale for these strains was based on their genome availability ${ }^{22-24}$, knowledge of their host interaction capacity ${ }^{25,26}$, their robustness and growth capacity ${ }^{22,24,26}$ (Extended Data Figure 2a), in addition to information on their safety in humans after oral ${ }^{27,28,29}$, nasal ${ }^{30}$ and vaginal ${ }^{31}$ high-dose application. L. rhamnosus GG was also selected because of previous reports on its capacity to inhibit the toxic effects of $S$. aureus on epidermal keratinocytes ${ }^{32}$, its strain-dependent capacity to promote re-epithelialization ${ }^{33}$ and to augment tight-junction barrier function in human primary epidermal keratinocytes ${ }^{34}$, and our previous experience with this probiotic strain ${ }^{25}$. For microbiome modulation, $C$. acnes was targeted as model pathobiont associated with the inflammatory character of acne vulgaris. S. aureus was also targeted as an important pathogen causing skin inflammation. When the activity of spent culture 
126 supernatant of our collection of Lactobacillus strains was screened for antimicrobial effects on the

127 growth of $C$. acnes in suspension, all Lactobacillus strains tested inhibited the growth of $C$. acnes

128 ATCC6919 and S. aureus ATCC29213, but L. pentosus KCA1 (vaginal origin) and L. plantarum WCFS1

129 (saliva origin) were among the bacteria tested able to exert the highest inhibition (Figure $\mathbf{2 b}$ and

130 Extended Data Figure 2b). Other related strains tested, including Staphylococcus epidermidis 12228,

131 did not inhibit $C$. acnes growth (Extended data Figure 2). In addition, these strains were able to

132 significantly reduce the lipase activity of $C$. acnes (Figure 2c). These lipase enzymes are involved in

133 inflammation of the skin induced by $C$. acnes, because they metabolize sebum into free fatty acids

134 which may lead to skin irritation ${ }^{5}$. Furthermore, because lactic acid has a strong antimicrobial

135 activity ${ }^{35}$, as well as a documented dose-dependent capacity to ameliorate the appearance of

136 keratoses and acne in dermatology ${ }^{36}$, we also substantiated lactic acid production by the selected

137 lactobacilli (Figure 2d). Furthermore, we validated that the three selected lactobacilli did not exhibit

138 toxic or overt inflammatory responses on primary skin cells (Figure 2e), in agreement with genome

139 predictions $\mathrm{s}^{22-24}$ and laboratory validation of antibiotic resistance profiles according to the guidelines

140 of the European Food Safety Authority (EFSA) ${ }^{37}$.

142 Viable Lactobacillus formulation in $\mathrm{O} / \mathrm{W}$ cream. We then aimed to design a topical formulation

143 suitable for the application of live bacteria in a sufficient dose on the skin. The selected bacteria were

144 freeze-dried for stability reasons ${ }^{38}$ and embedded in the core of 2-compartment microcapsules

145 (Figure 3a). Various processing conditions were optimized as described in the Methods section and

146 schematized in Figure 3a, resulting in capsules of $1500-2000 \mu \mathrm{m}$ diameter with a core of suspended

147 freeze-dried bacteria that can be released upon applying mechanical pressure, such as rubbing on

148 the skin (Figure 3b). Ingredients were selected so that they did not significantly impact on the growth

149 capacity of the skin commensals and pathobionts (tested for S. epidermis, S. aureus, and L. crispatus)

150 (Extended Data Figure $2 \mathrm{c}-\mathrm{d}$ ). This formulation and encapsulation approach significantly improved 
151 the viability for storage at $4^{\circ} \mathrm{C}$ and even at $25^{\circ} \mathrm{C}$, compared to non-encapsulated freeze-dried bacteria

152 when suspended in a carrier oil-in-water (O/W) cream (Figure 3c) and this for up to 6 months (Figure

153 3d).

154 Subsequently, the skin irritation potential was checked for 20 volunteers with skin patch tests

155 according to Basketter et al..$^{39}$. No erythema, dryness or edema was observed in any of the

156 volunteers studied (skin irritation index: 0.00) (Extended Data Table 2). For comparison, adapalene

157 products, which are naphthoic acid derivatives with retinoid activity and documented efficacy in the

158 treatment of mild-to-moderate acne vulgaris, have a mean cumulative irritation index between 0.25 -

$159 \quad 1^{40}$. Also the widely used combined clindamycin-benzoylperoxide treatment for moderate acne has

160 been reported to frequently induce dry skin, flaky/peeling skin, irritated skin, itchy skin and redness

161 in acne patients ${ }^{41}$.

Lactobacillus skin microbiome modulation. Subsequently, we applied the topical cream twice daily in an open-label 'proof-of-concept' trial to ten volunteers for eight weeks twice daily at $10^{8} \mathrm{CFU}$ per application ( \pm 1 gram/application) (Figure 4a). Patients with mild-to-moderate acne symptoms that were not using antibiotics or another acne treatment were included by the responsible dermatologist (Extended Data Table 3). The impact of the Lactobacillus cream on their facial skin microbiome was monitored by $16 \mathrm{~S}$ amplicon sequencing at four different time points, over a period of 10 weeks (Figure 4a). In this way, the skin baseline microbiome before, during and after the treatment was compared. The skin acne microbiome of these patients at the time of inclusion was especially

171 characterized by an increased relative abundance of Staphylococcus taxa $(p=0.0058$, Wilcoxon rank 172 sum test) when compared to the healthy controls (Figure 4b) (Extended Data Figure 4 for 3 specific 173 Staphylococcus ASVs). No significant difference in relative abundance of Lactobacillus taxa was 174 observed between our patients and the reference samples at time of inclusion (Extended Data 
176 salivarius, a taxon also belonging to the lactic acid bacteria with lactic acid production as core

177 function (Extended Data Figure 4). After application of the cream with the lactic-acid producing

178 lactobacilli, the facial skin samples of our acne patients at visit 2 and visit 3 clearly clustered

179 separately on a PCoA plot (Figure 4c). Interestingly, in 7 of 10 patients at visit 2 (4 weeks) and 8/10

180 patients at visit 3 (8 weeks), Lactobacillus ASVs were found in relative high abundances (between

$18120.9 \%$ and $92.8 \%$ ), while in three patients at visit 2 and two patients at visit 3 , their relative

182 abundance was below 5\% (between 0.015 and $1.1 \%$ ) (Figure 4d and Extended Data Figure 3). ASV

183 analysis via EZ $\operatorname{taxon}^{42}$ and comparison with the whole genome sequences ${ }^{22-24}$ for rRNA copy variants

184 confirmed that the detected ASVs matched the applied lactobacilli. Interestingly, the three probiotic

185 strains appeared to persist on the skin in similar numbers (Figure 4d). To substantiate that the

186 lactobacilli detected on the skin were still viable, samples were also plated on Lactobacillus-selective

187 MRS agar. Most samples at visit $2(7 / 9)$ and visit $3(6 / 7)$ were culture-positive, indicating that - at

188 least some of- the lactobacilli applied were metabolically active on the skin (Figure 4d). At visit 4 (two

189 weeks after the stop of the treatment), most Lactobacillus ASVs had disappeared and also growth in

190 MRS medium was markedly reduced, further substantiating that the lactobacilli detected originated

191 from the applied topical cream. We then explored whether the presence of lactobacilli during

192 treatment had impacted on the pathobionts of acne ( $C$. acnes and Staphylococcus taxa). The relative

193 abundance of both pathobiont taxa dropped indeed at visit 2 and 3 and increased again at visit 4 ( $p<$

1940.05 for visit 3 versus visit 1 - Wilcoxon test for Staphylococcus) (Figure $\mathbf{4 b}$ ).

195 Lactobacillus improvement of acne symptoms. Subsequently, the acne symptoms were clinically

196 scored as the presence of inflammatory lesions and comedones. This analysis showed an overall

197 improvement of the acne symptoms in all patients treated with the Lactobacillus cream, as reflected

198 by a significant reduction in inflammatory lesions at visit 2 and 3 compared to visit 1 , and a significant

199 reduction in comedone counts at visit 2 (Figure 5a). A significant association between comedonal

200 counts and both Staphylococcus and Propionibacterium/Cutibacterium was also found (Figure 5b),

201 but not for the inflammatory lesions (Extended Data Figure 5). Of note, when the treatment 
stopped, the acne scores increased again, indicating that the applied lactobacilli and associated

203 microbiome - staphylococcal modulation - did not persist, in agreement with the fact that the

204 exogenously applied lactobacilli could not permanently colonize (Figure $\mathbf{4} \mathbf{d}$ ). On the other hand, this

205 increase in acne scores when the Lactobacillus application stopped, further suggests a possible causal

206 association between the applied lactobacilli and the acne symptom reduction.

209 Acne vulgaris is a common reason for long-term antibiotic use, with dermatologists prescribing 210 antibiotics more commonly than any other physician group ${ }^{7}$. Here, we applied a multiphasic and 211 multidisciplinary approach to substantiate that Lactobacillus strains have potential as skin probiotics 212 to target acne. First, we provided detailed information that lactobacilli (and other lactic acid 213 producing taxa) are unneglectable endogenous members of the human skin microbiota, with relative 214 abundances in between those of human vaginal ${ }^{43}$ and stool $^{10}$ samples. Of interest, phylogenetic 215 placement of the Lactobacillus sequences detected in our data (amplicon sequence variants) and the 216 curatedMetagenomicData recently described by Pasolli et al. ${ }^{13}$ showed that the dominant 217 Lactobacillus taxa (L. crispatus, L. iners, L. gasseri, L. jensenii) of the vaginal community are also 218 among the most prevalent Lactobacillus taxa for the skin. Previous studies have briefly acknowledged 219 the presence of lactobacilli in the skin microbiota ${ }^{44,45}$, however such detailed analysis of specific 220 Lactobacillus taxa in the skin niche had not yet been performed. Yet, we also showed that to apply 221 selected lactic acid bacteria on the skin, other properties such as robustness to (processing) stress 222 conditions and growth capacity are required, in addition to safety and lack of (transferable) antibiotic 223 resistance properties (as rationalized in Figure 2a). Following this rationalized scheme, we did 224 manage to translate our results directly from in vitro lab tests with skin cells and pathogens to human 225 volunteers, without the need for animal testing. Spent-culture supernatant of the selected $L$. 226 rhamnosus GG, L. plantarum WCFS1 and L. pentosus KCA1 could inhibit the growth of $C$. acnes and S. 
227 aureus in vitro, could survive the formulation in capsules in an O/W cream and were found in similar

228 amounts after 1/1/1 application on the facial skin of patients with mild-to-moderate acne symptoms.

229 Twice daily topical application of this cream with the live lactobacilli was able to reduce inflammatory

230 acne lesions and comedone formation in the ten patients included in the open-label pilot study, and

231 was associated with a reduction in Staphylococcus relative abundance (as summarized in Figure 6).

232 Our 16S rRNA ASV-based comparison of the acne facial microbiome of 30 healthy volunteers and 27

233 patients with acne symptoms suggests indeed that Staphylococcus taxa are increased in acne

234 patients and that Staphylococcus could thus form an interesting acne target to further investigate.

235 ASV level analysis of the sequenced V4 region of the 16S rRNA gene did not allow identification of the

236 Staphylococcus taxa up to species/strain level, so that no distinction between S. epidermidis and S.

237 aureus and specific more virulent strains could yet be made. On the other hand, microbiome

238 comparison of the skin of subjects with a healthy skin and patients with mild-to-moderate acne

239 vulgaris also pointed to other lactic acid producing bacteria such as Streptococcus salivarius as being

240 potentially beneficial against acne.

241 Our findings are consistent with the growing body of evidence that lactic acid bacteria such as

242 lactobacilli can be applied at multiple human body sites to target the microbiome, epithelial barrier

243 function and immune system in various conditions ${ }^{35}$. In this study, we now add support for the skin

244 as topical therapeutic area. Evidently, compliance of this probiotic therapy by the patients will be a

245 key aspect to monitor - and possibly improve- in the future. The promising results from our proof-of-

246 concept study with live lactic acid-producing microbes should now also be confirmed in larger-scale

247 and longitudinal studies, in addition to more molecular studies towards underlying antimicrobial and

248 anti-inflammatory mechanisms and probiotic effector molecules (Figure 6). Together, these studies

249 will contribute to a new era of skin therapeutics based on microbiome modulation, as well as more

250 fundamental and mechanistic insights on the keystone core functions of lactic acid bacteria for skin

251 health. 


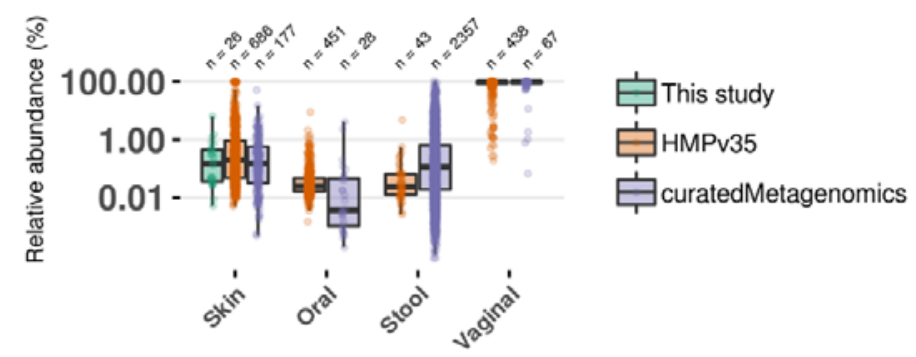

$\mathbf{a}_{\mathrm{s}}$

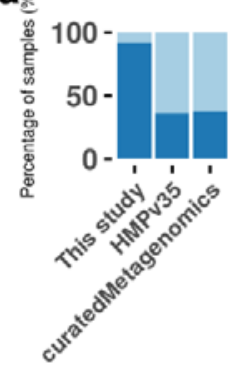

C b
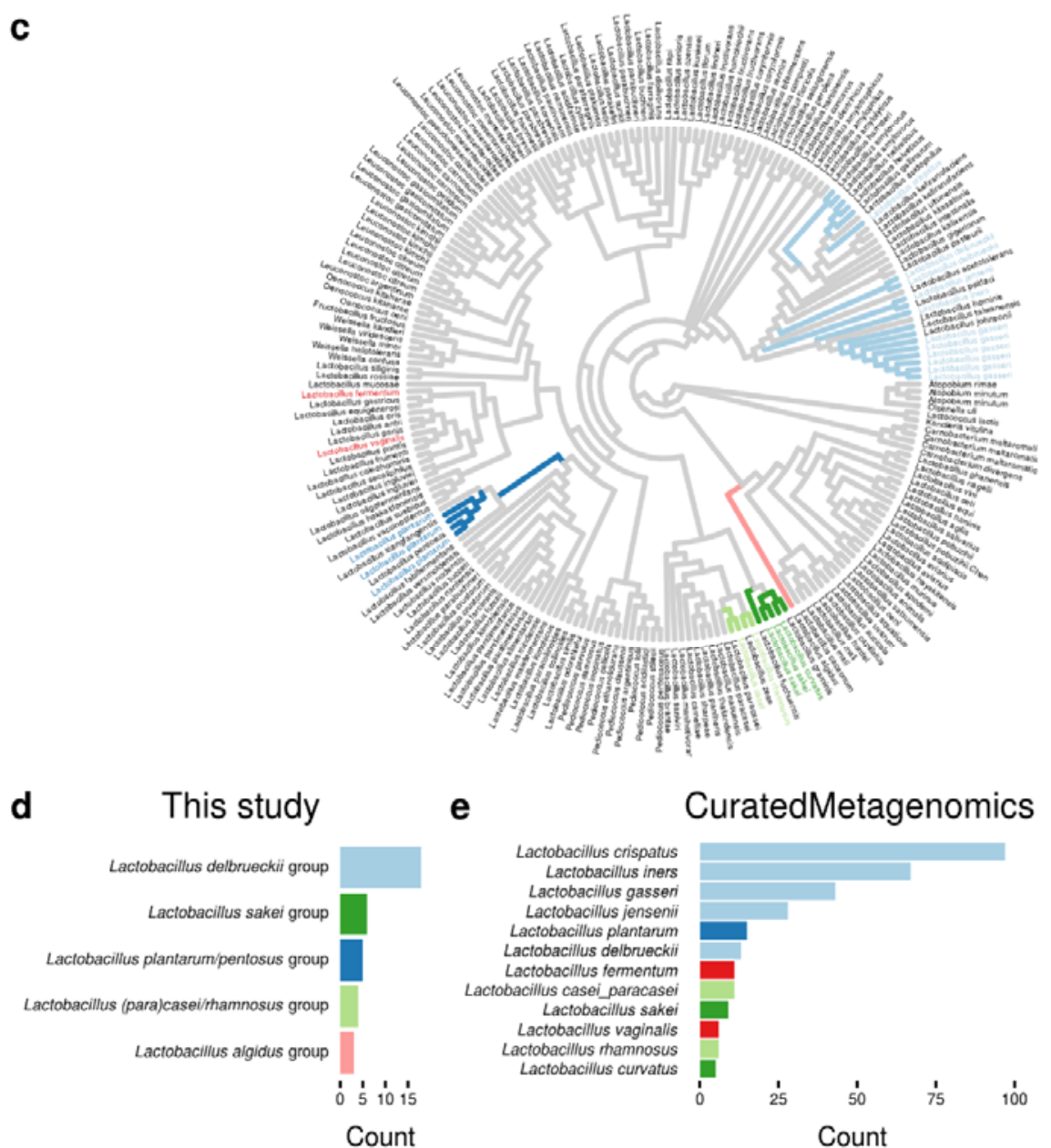

e

CuratedMetagenomics

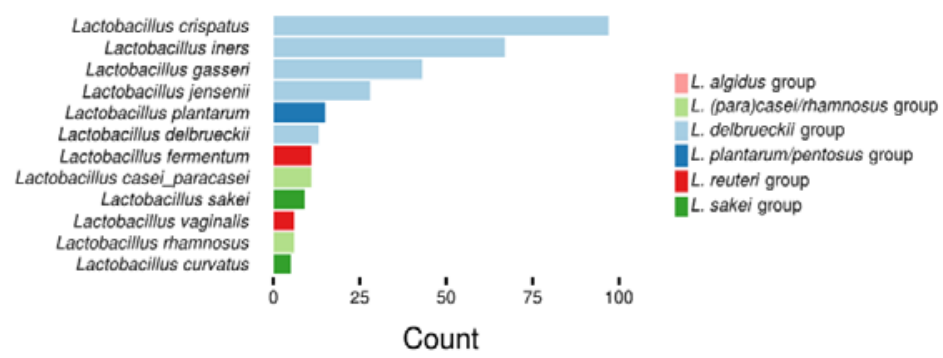

Figure 1 - Lactobacillus taxa in skin samples of $16 \mathrm{~S}$ rRNA amplicon and shotgun metagenomic data

(a) Presence and absence of Lactobacillus in skin samples of this study, the Human Microbiome Project (HMPv35) ${ }^{46}$ and shotgun metagenomic datasets from three studies ${ }^{14,15}$ and 16, accessed through the curatedMetaganomics R Package. (b) Comparison of relative abundance of Lactobacillus in different niches of the three used datasets. The $y$-axis is represented in log scale. (c) 165 rRNA cladogram of the Lactobacillus Genus Complex. Branches are colored based on phylogenetic placement of Lactobacillus ASVs from this study and the phylogenetic group (as described by Duar et $a l^{19}$ ) they belong to. Tip labels are colored based on the 12 most abundant Lactobacillus species found in the skin shotgun metagenomic datasets. ( $d$ and e) The most abundant Lactobacillus members in this study (d) and the skin shotgun metagenomic datasets (e) colored according to the phylogenetic group of the Lactobacillus Genus Complex they belong to. 

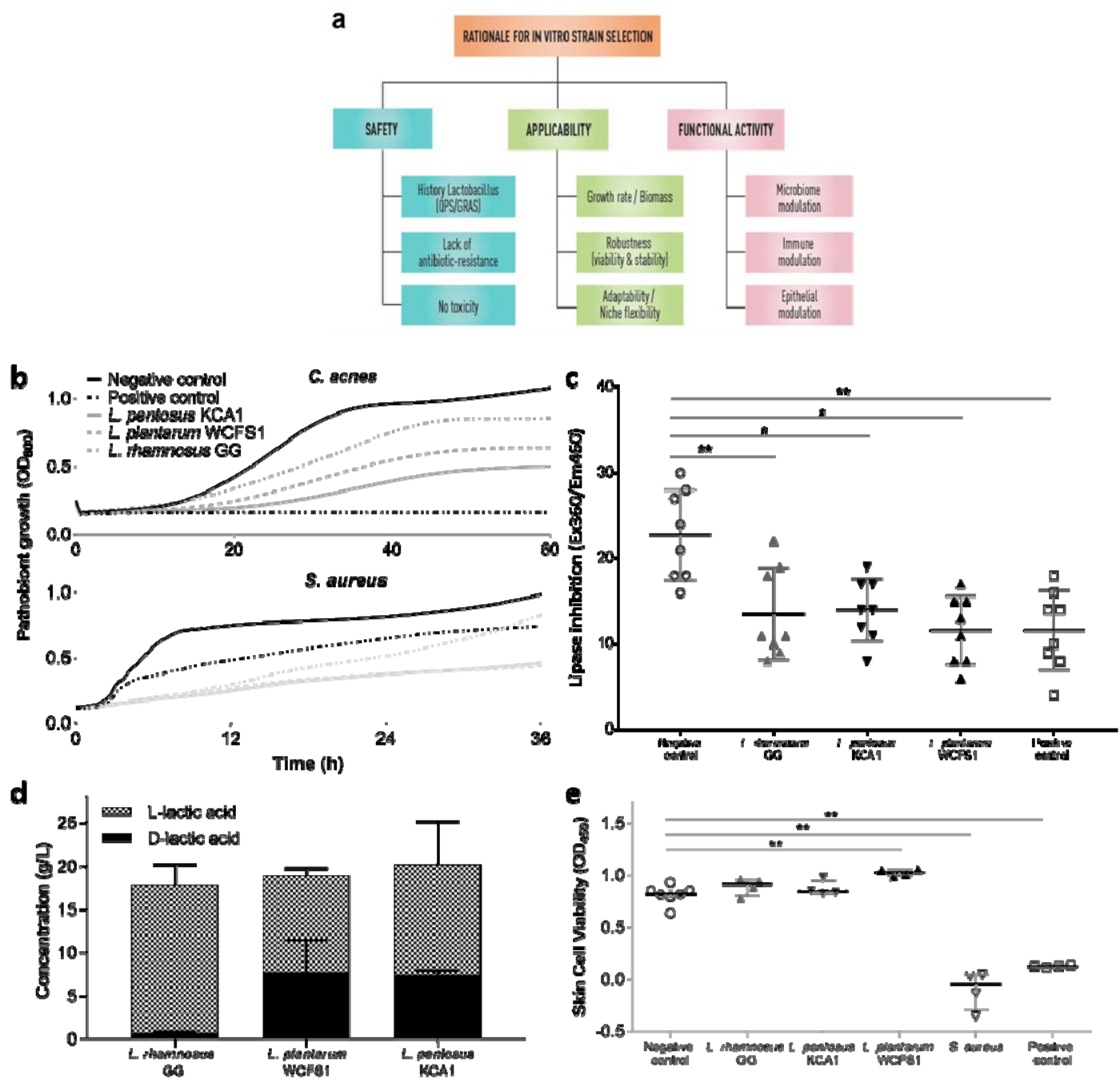

Figure 2 - In vitro selection of Lactobacillus strains for targeted application against acne vulgaris.

(a) Schematic overview of the rationale for the selection. Each criterion needs to be taken into account upon selection. Laboratory or genomic prediction tests exist for each criterion. More information can be found in the main text. (b) Antimicrobial activity of the spent-culture supernatant of the selected Lactobacillus strains against the two pathobionts tested, $C$. acnes and S. aureus, and compared to the positive control $(10 \mathrm{mg} / \mathrm{mL}$ Clindamycin, a common antibiotic used in acne). MRS at $\mathrm{pH} 4$, which is comparable to the $\mathrm{pH}$ of the spent supernatant of lactobacilli, was used as a negative control. (c) Inhibition of lipase activity of $C$. acnes by the spent-culture supernatant of the selected Lactobacillus strains, and compared to the positive control $(10 \mathrm{mg} / \mathrm{mL}$ Clindamycin) and the negative control (MRS). (d) Concentration of L-lactic acid and D-lactic acid as key antimicrobial and skinmodulating molecules produced by the selected lactobacilli after overnight incubation in MRS broth. (e) Skin cell viability results of NHEK cells after addition of the selected lactobacilli compared to the negative control, keratinocyte growth medium 2, and positive controls, S. aureus and Triton-X, measured at $450 \mathrm{~nm}$ using an XTT assay. Statistical analysis were performed using a Mann-Whitney test where ${ }^{*}=p<0.05$ and ${ }^{* *}=p<0.01$. 
A

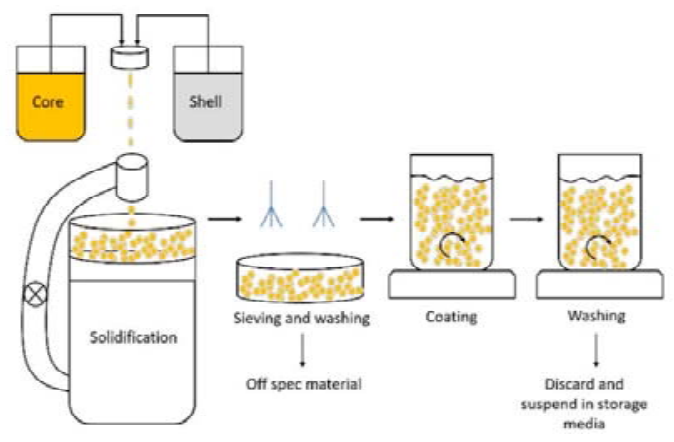

C

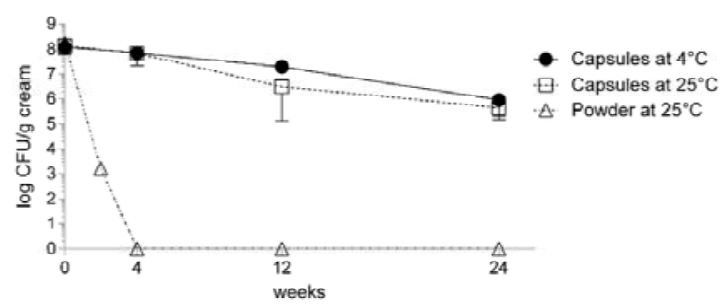

B

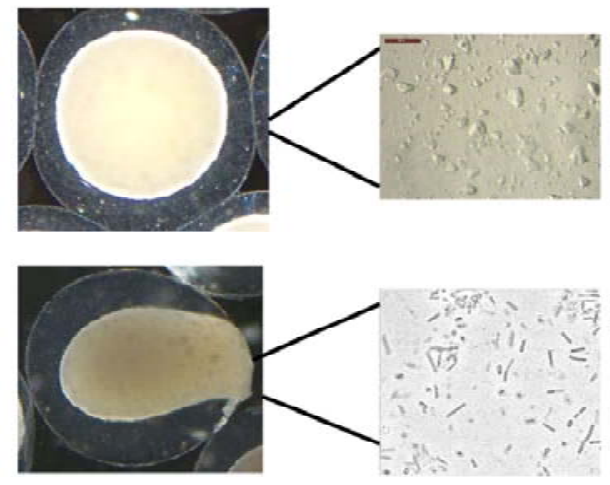

D

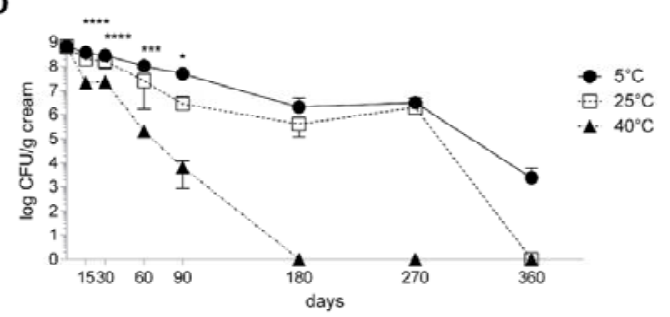

Figure 3 - Formulating live lactobacilli in a topical cream

(a) Schematic representation of the micro-encapsulating process with the bacteria in the core suspension and an outer shell made by the shell solution. (b) Resulting micro-capsules with a core of freeze-dried bacteria suspended in oil compared to microcapsules in which a force is applied just before application on the skin, hereby releasing the bacteria and activating them through wateruptake. (c) Survival of the bacteria in the microcapsules after different days compared to nonencapsulated freeze dried bacterial powder in an O/W cream. (d) Survival of the encapsulated bacteria in o/w cream tested according to the International Council for Harmonisation of Technical Requirements for Pharmaceuticals for Human Use Q1A(R2). Statistical analysis was performed using a Two-way ANOVA where $*=p<0.05,{ }^{* * *}=p<0.001$ and ${ }^{* * * *}=p<0.0001$. 


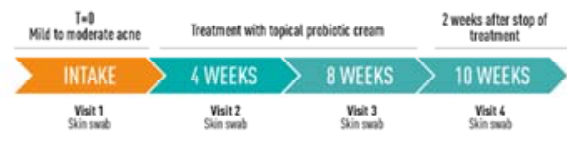

b

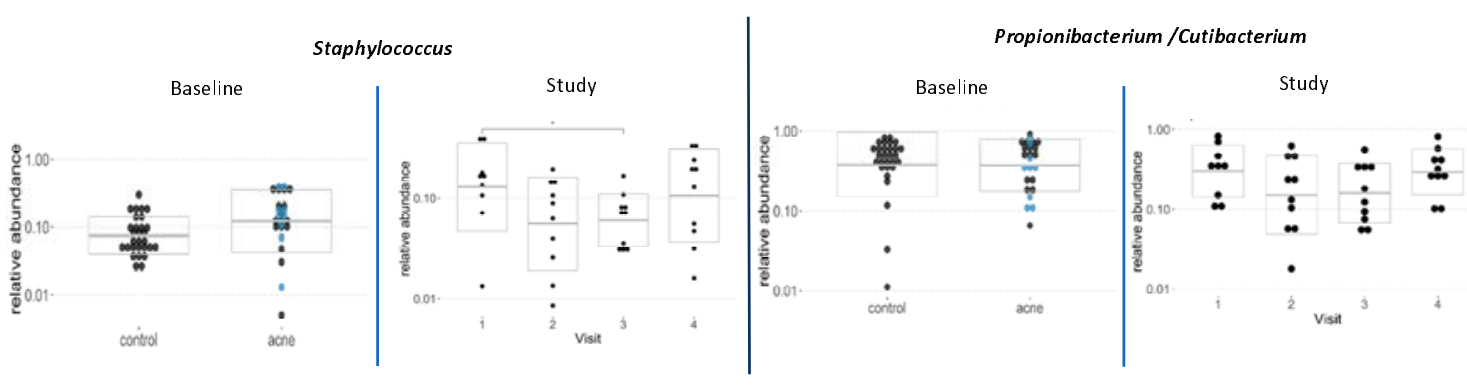

d

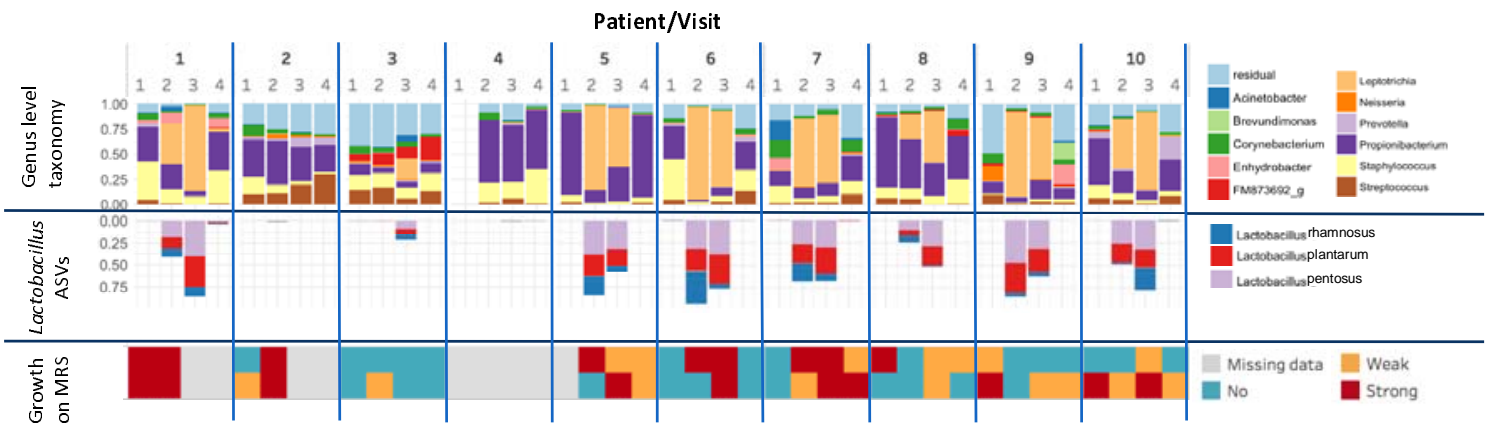

Figure 4 - Impact of the Lactobacillus cream on the skin microbiome

(a) Schematic overview of the POC intervention study with the O/W cream containing the selected and formulated lactobacilli, the visits at which a skin swab was taken and dermatological symptom analysis was performed by the dermatologist. The cream was applied twice daily for 8 weeks with a minimal dose of $10^{6} \mathrm{CFU} /$ application. (b) Relative abundance of Staphylococcus and

Propionibacterium/Cutibacterium respectively at baseline and over the four visits of the study (right). For the baseline, skin samples of the 30 healthy volunteers without acne symptoms (cfr. Figure 1) and 27 patients with mild-to-moderate acne symptoms were compared. Of these 27 acne patients, 10 patients (indicated with blue dots) were included in the Lactobacillus intervention Study

(Study) shown at the right side of each panel. For the study visits, $p<0.05$ for visit 3 versus visit 1 based on Wilcoxon ranks test is indicated with a star. (c) PCOA plot distributing samples according to beta-diversity (Bray-Curtis distance). Similar samples are located closely to each other, and colored by visit. (d) Microbial communities during the study period with the genus-level taxonomy indicated

(top), relative abundance of the three Lactobacillus ASVs resulting from the cream (middle) and observed growth on MRS medium (top row on agar, bottom row growth in MRS broth) after addition of the skin samples (bottom). Other Lactobacillus ASVs were not observed at a higher relative abundance than $1 \%$. Samples were ordered by participant and by visit. 
a

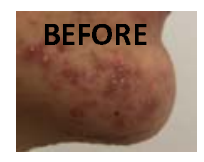

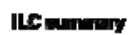

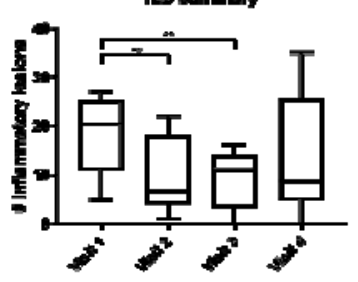

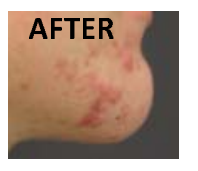

Coming

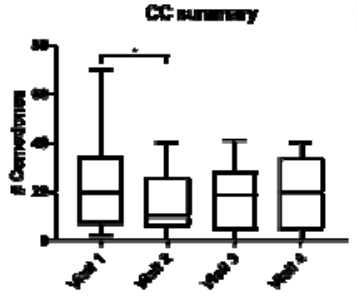

b

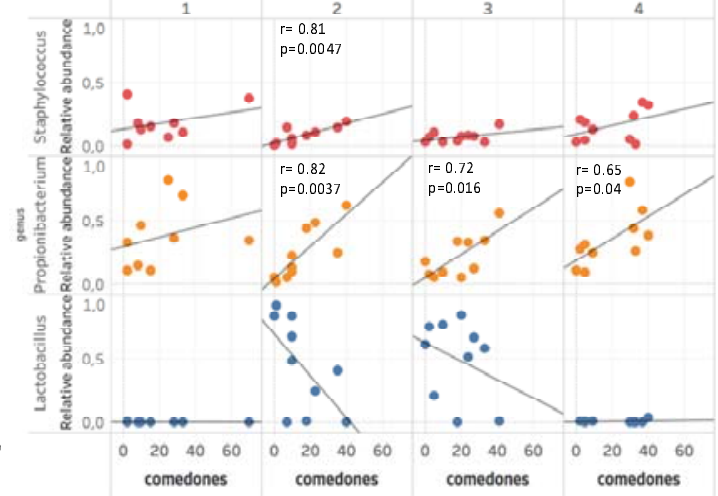

Figure 5- Effect of the Lactobacillus cream on acne symptoms and correlation with microbiome data

(a) Evolution of counts of inflammatory lesions (left) and comedones (right) over the course of the study, grouped by visit. All 10 patients included in the pilot study showed a clinical improvement after the application of the cream as exemplified with a picture of the acne spot area of one patients at visit 1 versus visit 2 . Statistical analysis were performed using a Wilcoxon matched-pairs signed rank test where $*=p<0.05$ and ${ }^{* *}=p<0.01$ (b) Correlation of relative abundances of Staphylococcus (top), Propionibacterium/Cutibacterium (middle) and Lactobacillus (bottom) to comedonal counts, per visit. Pearson correlation coefficient and $p$-values are indicated where $p<0.05$. 


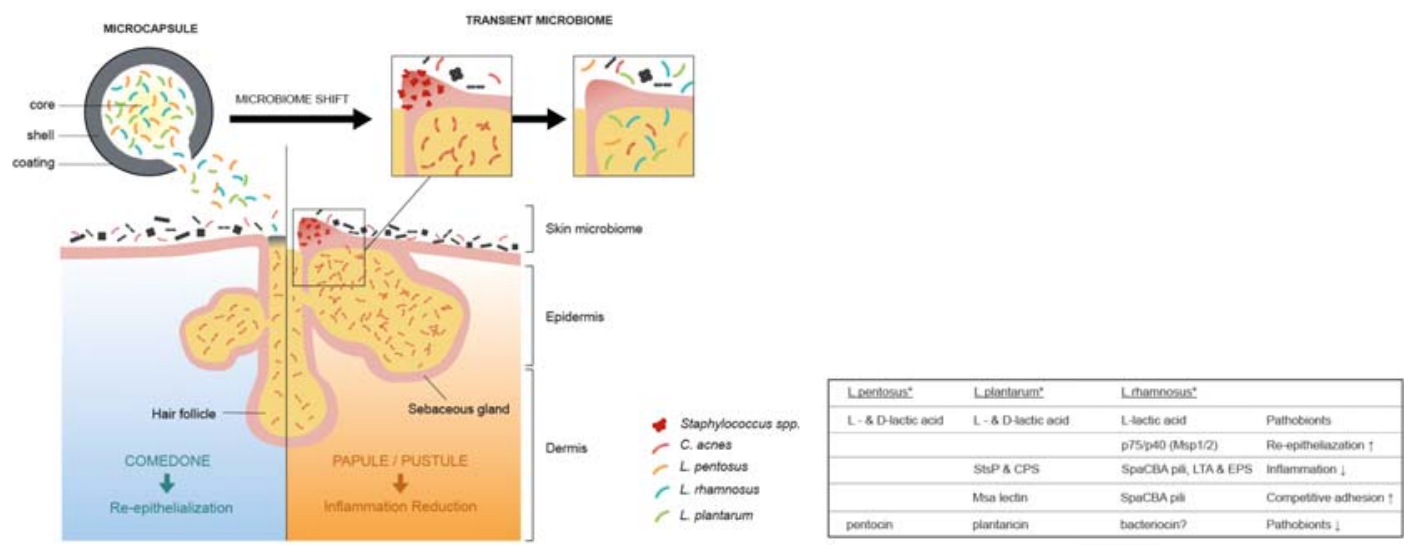
in a topical cream modulate skin microbiome and improve acne symptoms.

Specific Lactobacillus strains were selected and formulated in capsules in an oil (O) in water (W) cream that release the probiotics upon rubbing on the skin. Microbiome analysis (16S amplicon sequencing), as well as counting of comedone and inflammatory lesions substantiated that these lactobacilli could reduce inflammation and comedone formation, as well have transient impact on the skin microbiome, especially by decreasing the relative abundance of staphylococci and $C$. acnes as acne pathobionts. The postulated mode of action (also indicated with * in table) includes their antimicrobial activity against pathobionts by lactic acid (this study), competitive exclusion ${ }^{47,32}$ and possibly bacteriocins ${ }^{48,24}$, their capacity to reduce inflammation, e.g. by the serine-threonine rich protein StsP of $L$. plantarum WCFS $1^{49}$ or SpaCBA pili of $L$. rhamnosus $\mathrm{GG}^{50,51}$, and their capacity to promote re-epithelialization $^{33,34}$, by e.g. the secreted proteins Msp1 (p75)/Msp2 (p40) for $L$. rhamnosus $\mathrm{GG}^{29,52}$. Yet, the involvement of these probiotic effector molecules remains to be further substantiated in follow-up work. 
Bacterial growth. Lactobacillus strains were grown at $37^{\circ} \mathrm{C}$ in de Man, Rogosa and Sharpe (MRS) medium (BD Difco, Erembodegem, Belgium). Propionibacterium acnes ATCC6919 was inoculated in reinforced clostridial broth (LabM Limited, Heywood, UK), supplemented with $0.2 \%$ Tween 20 and cultured microaerobically $\left(5 \% \mathrm{CO}_{2}\right)$ at $37^{\circ} \mathrm{C}$. Staphylococcus aureus ATCC29213 was grown in MuellerHinton broth at $37^{\circ} \mathrm{C}$. Solid media contained $1.5 \%(\mathrm{w} / \mathrm{v})$ agar. Time-course experiments were also performed analysing the antimicrobial activity of spent culture supernatant (SCS) of the selected Lactobacillus strains against C. acnes and S. aureus ATCC29213 (cfr. ${ }^{53}$ ). Additionally, the impact of this SCS $(10 \%)$ on the lipase activity of $C$. acnes was determined as previously described ${ }^{5}$. to manufacturer's recommendations in Keratinocyt Growth medium 2 (Promocell, Heidelberg, Germany). Cytotoxicity of probiotic strains was assessed using the 2,3-Bis(2-methoxy-4-nitro-5sulfophenyl)-2H-tetrazolium-5-carboxanilide (XTT, Sigma-Aldrich) cell viability assay. NHEK cells were seeded at a density of 5000 cells/well in $96-$ well plates and cultured until confluent. Overnight

352 CFU/well and incubated for $2 \mathrm{~h}$ at $5 \% \mathrm{CO} 2$ and $37^{\circ} \mathrm{C}$. Triton $\mathrm{X}-100(0.5 \%)$ was used as a positive 353 control. brushing the cheek (control group) or the affected area on the face (patients) with a FloqSwab (Copan) over an area of $\pm 10 \mathrm{~cm}^{2}$ or around the lesions. Swabs were then transferred to a falcon containing $800 \mu \mathrm{l}$ Bead solution of QIAamp PowerFecal DNA kit (Qiagen). Samples were stored at $4^{\circ} \mathrm{C}$ until further processing (maximally 14 days). Before DNA extraction, samples were vortexed for 1 DNA extraction were executed according to manufacturer's instructions. 
361 Illumina MiSeq 16S rDNA gene amplicon sequencing. The primers used for Illumina MiSeq

362 sequencing were based on the previously described $27 \mathrm{~F}-338 \mathrm{R}$ or $515 \mathrm{~F}-806 \mathrm{R}$ primers ${ }^{54}$ and altered for

363 dual-index paired-end sequencing, as described earlier ${ }^{55}$ (Extended Data Table 4). Separate runs

364 were carried out for V1V2 and V4 rRNA gene variable regions. Quality control and processing of reads

365 was performed using the R package DADA2, version $1.6 .0^{56}$. Denoised reads (amplicon sequence

366 variants or ASVs) were merged and read pairs with one or more conflicting bases between the

367 forward and reverse read were removed. Chimeric sequences were removed using the function

368 "removeBimeraDenovo". Finally, ASVs were classified from the kingdom to the genus level using the

369 EzBioCloud 165 database ${ }^{42}$. A species annotation was added to each ASV by listing the species of all

370165 sequences in the database that showed an exact match to the ASV sequence. Contaminants

371 were identified using the approach of Jervis-Bardy et $a l^{57}$. ASVs with a strong negative correlation

372 between relative abundances and total sample read counts were considered contamination. For each

373 ASV, this correlation was calculated and tested for significance. ASVs with a p-value less than 0.0001

374 were removed. Samples were filtered by removing those with less than 1000 reads left after all read

375 and ASV filtering steps.

376 Biostatistical and bioinformatics analysis. Processing of the ASV table, ASV annotations (e.g.

377 classification) and sample annotations (metadata) were performed using the in-house $\mathrm{R}$ package

378 "tidyamplicons", publicly available at github.com/SWittouck/tidyamplicons. For the analyses at the

379 genus level, ASV read counts were aggregated at the genus level or, if unavailable, at the most

380 specific level at which taxonomic annotation was available.

381 Analysis of public datasets. Processed OTU-table and sample metadata from the Human Microbiome

382 Project (HMPv35) ${ }^{46}$ and the shotgun metagenomic datasets were retrieved using the MicrobeDS R

383 package and curatedMetagenomics $\mathrm{R}$ package ${ }^{16}$ respectively. All data was loaded, processed and

384 visualized in the R-environment using Phyloseq. All scripts are available at

385 https://github.com/LebeerLab/skin_acne_study. 
qPCR for estimation of absolute bacterial concentrations. QPCR was performed in duplicate on a 20fold dilution (to avoid interference of PCR inhibitors) of total DNA isolated from the samples, using the StepOnePlus real time qPCR system (Applied Biosystems ${ }^{\oplus}$, Foster City, California, USA), SYBR ${ }^{\oplus}$ Green chemistry (PowerUp $^{\mathrm{TM}}$ SYBR $^{\oplus}$ Green Master Mix, Applied Biosystems ${ }^{\oplus}$, Foster City, California, USA) and primers as indicated in Extended Data Table 4. Standard curves were used to estimate bacterial concentrations in the samples and derived from serially diluted DNA from an overnight culture of Lactobacillus crispatus LMG12005 isolated similarly as the samples. Bacterial concentration was determined by plating.

Formulation of lactobacilli in microcapsules and $\mathrm{O} / \mathrm{W}$ cream. A single colony of the three selected probiotic strains was grown until stationary phase and lyophilized. The lyophilized bacterial powder was grinded and milled (Frewitt, Switzerland) to obtain a fine powder ( $\pm 10^{11} \mathrm{CFU} / \mathrm{gram}$ ) and subsequently encapsulated via a core-shell encapsulation approach. Briefly, the strains were mixed in equal amounts and homogeneously suspended to obtain a stable oil-based feed core suspension. The shell feed solution contained a hydrocolloid alginate polymer as gelling agent. Both liquid feeds were pumped to a concentric nozzle, to obtain a concentric fluid flow. The laminar liquid flow was broken up by a vibrational unit to obtain spherical -droplets that were solidified upon falling in a calcium-based solidification solution, forming the capsules. The collected capsules $\left(10^{9}-10^{10}\right.$ CFU/gram) were washed and suspended in an oil-in-water cream containing the following ingredients: Aqua, Glycerin, Polyglyceryl-3 Rice Branate, Propylheptyl Caprylate, Cetyl Alcohol, Nylon 6/12, Caprylic/Capric Triglyceride, Squalane, PEG-400, Cyclopentasiloxane, Cetearyl Alcohol, Prunus Amygdalus Dulcis Oil, Allantoin, Limnanthes Alba Seed Oil, Tocopheryl Acetate, Xanthan Gum, Cyclohexasiloxane, Helianthus Annuus Seed Oil, Algin, p-Anisic Acid, Silica Dimethyl Silylate, Disodium EDTA, Sodium Hydroxide, Hydrochloric Acid. The ingredients of this cream, mainly the emulsifiers and preservatives, were selected to be compatible with the micro-capsules and bacteria, both during storage and upon release of the probiotics. Hereto, the impact of the topical cream without the capsules on the growth of four skin reference bacteria (S. aureus, S. epidermidis, L. crispatus and C. 
412 acnes) was evaluated at a concentration of 1,10 and $100 \mathrm{mg} / \mathrm{ml}$, by a time-course analysis of OD600

413 measurements as described above. Mechanical force (rubbing on the skin) was confirmed to break

414 the capsules, releasing the inner core material containing the suspended probiotics. Skin irritation

415 tests with the O/W cream containing the freeze -dried lactobacilli were performed as described

416 previously ${ }^{39}$.

417 Proof-of-concept human in patients with acne vulgaris. A proof-of-concept clinical trial was

418 performed on patients with mild-to-moderate acne vulgaris included after careful assessment of the

419 responsible dermatologist by counting of comedones and inflammatory lesions (Extended Data

420 Table 3). Patients were men between 12-25 years. Exclusion criteria were use of oral antibiotics

421 within 4 weeks prior to start of the study and use of systemic retinoids within 6 months prior to start

422 of study. Subjects provided written informed consent before the study began. Patients were asked to

423 apply the topical probiotic cream (containing $10^{8} \mathrm{CFU}$ of each Lactobacillus strain per application of 1

$424 \mathrm{~g}$ of the topical cream). Patients were asked to apply the cream twice daily for 56 days (8 weeks). The

425 patients were seen by a dermatologist at start (before the therapy) (visit 1), week 4 (visit 2), week 8

426 (visit 3) and week 10 (visit 4). A skin swab was taken at each visit, total DNA was extracted and

427 amplified for 165 amplicon sequencing as described above. Moreover, a clinical scoring was

428 performed and a photograph taken at each visit.

Clinical trial registration. The protocol of this study was in accordance with the Declaration of

431 Helsinki, and was approved by the ethics committee of the University Hospital of Antwerp (Belgium)

432 before initiation of the study. The study was given the approval number B300201628507 (Belgian

433 registration) and registered online at clinicaltrials.gov with unique identifier NCT03469076.

Data availability. Sequencing data are available at the European Nucleotide Archive with the accession number PRJEB27311. 
1. Grice, E. A. et al. Topographical and temporal diversity of the human skin microbiome. Science (80-. ). 324, 1190-1192 (2009).

2. Byrd, A. L., Belkaid, Y. \& Segre, J. A. The human skin microbiome. Nature Reviews

3. Hill, C. et al. Expert consensus document: The International Scientific Association for reclassification of selected species within the genus propionibacterium to the proposed novel genera acidipropionibacterium gen. Nov., cutibacterium gen. nov. and

6. Dréno, B. What is new in the pathophysiology of acne, an overview. Journal of the European

$$
\text { Academy of Dermatology and Venereology (2017). doi:10.1111/jdv.14374 }
$$

454 7. Del rosso, J. Q. et al. Status report from the scientific panel on antibiotic use in dermatology of the American acne and rosacea society part 1: Antibiotic prescribing patterns, sources of antibiotic exposure, antibiotic consumption and emergence of antibiotic resistance, impac. J. Clin. Aesthet. Dermatol. (2016). 
term use, biodiversity and proposals for specific and realistic safety assessments. FEMS Microbiology Reviews (2006). doi:10.1111/j.1574-6976.2006.00020.x

10. Heeney, D. D., Gareau, M. G. \& Marco, M. L. Intestinal Lactobacillus in health and disease, a driver or just along for the ride? Current Opinion in Biotechnology 49, 140-147 (2018).

11. Petrova, M. I., Lievens, E., Malik, S., Imholz, N. \& Lebeer, S. Lactobacillus species as biomarkers and agents that can promote various aspects of vaginal health. Frontiers in Physiology 6, (2015).

12. Mårtensson, A. et al. Effects of a honeybee lactic acid bacterial microbiome on human nasal symptoms, commensals, and biomarkers. Int. Forum Allergy Rhinol. 6, 956-963 (2016).

13. Pasolli, E. et al. Accessible, curated metagenomic data through ExperimentHub. Nature Methods 14, 1023-1024 (2017).

14. Chng, K. R. et al. Whole metagenome profiling reveals skin microbiome-dependent susceptibility to atopic dermatitis flare. Nat. Microbiol. 1, (2016).

15. Oh, J. et al. Biogeography and individuality shape function in the human skin metagenome. Nature 514, 59-64 (2014).

16. Tett, A. et al. Unexplored diversity and strain-level structure of the skin microbiome associated with psoriasis. npj Biofilms Microbiomes 3, 14 (2017).

17. Huttenhower, C. \& Human Microbiome Project Consortium. Structure, function and diversity of the healthy human microbiome. Nature (2012). doi:10.1038/nature11234

18. Wuyts, S. et al. Carrot juice fermentations as man-made microbial ecosystems dominated by lactic acid bacteria. Appl. Environ. Microbiol. (2018). doi:10.1128/AEM.00134-18

19. Duar, R. M. et al. Lifestyles in transition: evolution and natural history of the genus Lactobacillus. FEMS Microbiol. Rev. 41, S27-S48 (2017). 
20. Chu, D. M. et al. Maturation of the infant microbiome community structure and function across multiple body sites and in relation to mode of delivery. Nat. Med. 23, 314-326 (2017).

21. Banerjee, S., Schlaeppi, K. \& van der Heijden, M. G. A. Keystone taxa as drivers of microbiome structure and functioning. Nature Reviews Microbiology (2018). doi:10.1038/s41579-0180024-1

22. Kankainen, M. et al. Comparative genomic analysis of Lactobacillus rhamnosus GG reveals pili containing a human-mucus binding protein. Proc. Natl. Acad. Sci. U. S. A. 106, (2009).

23. Kleerebezem, M. et al. Complete genome sequence of Lactobacillus plantarum WCFS1. Proc. Natl. Acad. Sci. 100, 1990-1995 (2003).

24. Anukam, K. C. et al. Genome Sequence of Lactobacillus pentosus KCA1: Vaginal Isolate from a Healthy Premenopausal Woman. PLoS One 8, e59239 (2013).

25. Segers, M. E. \& Lebeer, S. Towards a better understanding of Lactobacillus rhamnosus GG host interactions. Microb. Cell Fact. 13, S7 (2014).

26. van den Nieuwboer, M., van Hemert, S., Claassen, E. \& de Vos, W. M. Lactobacillus plantarum WCFS1 and its host interaction: a dozen years after the genome. Microbial Biotechnology 9 , 452-465 (2016).

27. Tapiovaara, L. et al. Absence of adverse events in healthy individuals using probiotics analysis of six randomised studies by one study group. Benef. Microbes 7, 161-169 (2016).

28. van Baarlen, P. et al. Differential NF-kB pathways induction by Lactobacillus plantarum in the duodenum of healthy humans correlating with immune tolerance. Proc. Natl. Acad. Sci. (2009). doi:10.1073/pnas.0809919106

29. van Baarlen, P. et al. Human mucosal in vivo transcriptome responses to three lactobacilli indicate how probiotics may modulate human cellular pathways. Proc. Natl. Acad. Sci. U. S. A. 
108 Suppl, 4562-4569 (2011).

507

508

30. Skovbjerg, S. et al. Spray bacteriotherapy decreases middle ear fluid in children with secretory otitis media. Arch. Dis. Child. 94, 92-98 (2009).

31. Reid, G. \& Bruce, A. W. Selection of Lactobacillus Strains for Urogenital Probiotic Applications. J. Infect. Dis. 183, 77-80 (2001).

32. Mohammedsaeed, W., McBain, A. J., Cruickshank, S. M. \& O'Neill, C. A. Lactobacillus rhamnosus GG inhibits the toxic effects of Staphylococcus aureus on epidermal keratinocytes. Appl. Environ. Microbiol. 80, 5773-5781 (2014).

33. Mohammedsaeed, W., Cruickshank, S., McBain, A. J. \& O’Neill, C. A. Lactobacillus rhamnosus GG Lysate Increases Re-Epithelialization of Keratinocyte Scratch Assays by Promoting Migration. Sci. Rep. 5, (2015).

34. O'Neill, C. A., Sultana, R. \& McBain, A. J. Strain-dependent augmentation of tight-junction barrier function in human primary epidermal keratinocytes by lactobacillus and bifidobacterium lysates. Appl. Environ. Microbiol. 79, 4887-4894 (2013).

35. Sanders, M. E., Benson, A., Lebeer, S., Merenstein, D. J. \& Klaenhammer, T. R. Shared mechanisms among probiotic taxa: implications for general probiotic claims. Curr. Opin. Biotechnol. 49, (2018).

36. Tang, S. C. \& Yang, J. H. Dual effects of alpha-hydroxy acids on the skin. Molecules 23, (2018).

37. European Food Safety Authority. Guidance on the assessment of bacterial susceptibility to antimicrobials of human and veterinary importance. EFSA J. (2012). doi:10.2903/j.efsa.2012.2740.

38. Broeckx, G., Vandenheuvel, D., Claes, I. J. J., Lebeer, S. \& Kiekens, F. Drying techniques of probiotic bacteria as an important step towards the development of novel pharmabiotics. Int. 
J. Pharm. 505, (2016).

530

531

532

533

534

535

536

537

538

539

540

541

542

543

544

545

546

547

548

549

550

551

39. Basketter, D. A., Whittle, E., Griffiths, H. A. \& York, M. The identification and classification of skin irritation hazard by a human patch test. Food Chem. Toxicol. 32, 773-775 (1994).

40. Queille-Roussel, C. et al. Comparison of the cumulative irritation potential of adapalene gel and cream with that of erythromycin/tretinoin solution and gel and erythromycin/isotretinoin gel. Clin. Ther. 23, 205-212 (2001).

41. Feldman, S. R. \& Chen, D. M. How Patients Experience and Manage Dryness and Irritation From Acne Treatment. J Drugs Dermatol (2011).

42. Yoon, S. H. et al. Introducing EzBioCloud: A taxonomically united database of $16 \mathrm{~S}$ rRNA gene sequences and whole-genome assemblies. Int. J. Syst. Evol. Microbiol. (2017). doi:10.1099/ijsem.0.001755

43. Petrova, M. I., Lievens, E., Malik, S., Imholz, N. \& Lebeer, S. Lactobacillus species as biomarkers and agents that can promote various aspects of vaginal health. Front. Physiol. 6, (2015).

44. Zeeuwen, P. L. J. M. et al. Microbiome dynamics of human epidermis following skin barrier disruption. Genome Biol. (2012). doi:10.1186/gb-2012-13-11-r101

45. Li, X., Yuan, C., Xing, L. \& Humbert, P. Topographical diversity of common skin microflora and its association with skin environment type: An observational study in Chinese women. Sci. Rep. (2017). doi:10.1038/s41598-017-18181-5

46. Human, T. \& Project, M. Structure, function and diversity of the healthy human microbiome. Nature (2012). doi:10.1038/nature11234

47. Tytgat, H. L. P. et al. Lactobacillus rhamnosus GG Outcompetes Enterococcus faecium via Mucus-Binding Pili: Evidence for a Novel and Heterospecific Probiotic Mechanism. Appl. Environ. Microbiol. 82, 5756-5762 (2016). 
552

48. Diep, D. B., Straume, D., Kjos, M., Torres, C. \& Nes, I. F. An overview of the mosaic bacteriocin pln loci from Lactobacillus plantarum. Peptides (2009). doi:10.1016/j.peptides.2009.05.014

49. Remus, D. M., Kleerebezem, M. \& Bron, P. A. An intimate tête-à-tête - How probiotic lactobacilli communicate with the host. in European Journal of Pharmacology 668, (2011).

50. Lebeer, S. et al. Functional analysis of lactobacillus rhamnosus $\mathrm{GG}$ pili in relation to adhesion and immunomodulatory interactions with intestinal epithelial cells. Appl. Environ. Microbiol.

78, (2012).

51. Vargas García, C. E. et al. Piliation of Lactobacillus rhamnosus GG promotes adhesion, phagocytosis, and cytokine modulation in macrophages. Appl. Environ. Microbiol. 81, (2015).

52. Yan, F. et al. A lactobacillus rhamnosus GG-derived soluble protein, p40, stimulates ligand release from intestinal epithelial cells to transactivate epidermal growth factor receptor. $J$. Biol. Chem. 288, 30742-30751 (2013).

53. van den Broek, M. F. L. Multifactorial inhibition of lactobacilli against the respiratory tract pathogen Moraxella catarrhalis. Benef. Microbes 9, 429-439 (2018).

54. Caporaso, J. G. et al. Global patterns of $16 \mathrm{~S}$ rRNA diversity at a depth of millions of sequences per sample. Proc Natl Acad Sci USA 108, (2010).

55. Kozich, J. J., Westcott, S. L., Baxter, N. T., Highlander, S. K. \& Schloss, P. D. Development of a dual-index sequencing strategy and curation pipeline for analyzing amplicon sequence data on the MiSeq Illumina sequencing platform. Appl. Environ. Microbiol. 79, 5112-20 (2013).

56. Callahan, B. J. et al. DADA2: High-resolution sample inference from Illumina amplicon data. Nat. Methods 13, 581-583 (2016).

57. Jervis-Bardy, J. et al. Deriving accurate microbiota profiles from human samples with low bacterial content through post-sequencing processing of Illumina MiSeq data. Microbiome 
(2015). doi:10.1186/s40168-015-0083-8

577 Extended Data is available in the online version of this manuscript.

578 Acknowledgements. We kindly acknowledge Dr. K. Anukam for providing us Lactobacillus pentosus

579 KCA1. We thank Wannes Van Beeck, Dieter Vandenheuvel, Camille Allonsius, Leen Van Ham and all 580 other members of the ENdEMIC group for their assistance and/or fruitful discussions. We thank S. 581 Van Elslander for the help in the design of figures 2A, 4A and 6. We also greatly thank Dr. E. Pasolli 582 for the assistance with the CuratedMetagenomics Package. This work was funded by grants from the 583 Flanders Agency for Innovation \& Entrepreneurship (www.vlaio.be/en), including an IWT-SBO project 584 (IWT/50052) for the fundamental part of the research and a VLAIO R\&D project with Yun 585 Probiotherapy NV (formerly known as Axca Bvba) as SME partner for the applied part of the project. 586 The qPCR analyses were performed with a StepOne Plus (Applied Biosystems) machine funded by the 587 Fund for Scientific Research Flanders (1520114N). Sander Wuyts and Ilke De Boeck hold a personal $588 \quad$ PhD grant (IWT-SB 141198 and FWO 1S17916N respectively).

589 Author contributions. S.L., I.C., J.L. designed the study; J.L. clinically evaluated the patients and 590 collected patient samples; E.O. prepared the clinical and control samples for MiSeq sequencing with 591 the help of I.T. and I.D.B., Sa.Wu. did the shotgun metagenome analysis, Sa.Wu. St.Wi., and E.O. 592 analyzed sequence data. E.O., M.V.B., C.A. and I.S. did part of the microbiological and cell culture lab 593 experiments. I.C., T.H., F.K. formulated the lactobacilli in the topical cream. S.L. drafted the 594 manuscript and all authors approved the manuscript.

596 Author information. The authors declare the following competing interests. I.C. and T.H. were 597 employed at UAntwerp at the time of the study, but are currently working at the R\&D department of 598 Yun NV, a start-up company resulting from this research (www.yun.be). Based on the data presented 599 here, YUN NV has selected and formulated three Lactobacillus strains, $L$. pentosus YUN-V1.0, $L$. 
bioRxiv preprint doi: https://doi.org/10.1101/463307; this version posted November 19,2018 . The copyright holder for this preprint (which was not certified by peer review) is the author/funder, who has granted bioRxiv a license to display the preprint in perpetuity. It is made available under aCC-BY-NC-ND 4.0 International license.

600 plantarum YUN-V2.0 and L. rhamnosus YUN-S1.0 in their commercial ACN product. Part of the results

601 presented in this manuscript are included in patent applications PCT/EP2017/066176 and

602 PCT/EP2017/065006.

603 